\title{
Article \\ The Investigation on Ultrafast Pulse Formation in a Tm-Ho-Codoped Mode-Locking Fiber Oscillator
}

\author{
Jingcheng Shang ${ }^{1}$, Yizhou Liu ${ }^{1}$, Shengzhi Zhao ${ }^{1}$, Yuefeng Zhao ${ }^{2}$, Yuzhi Song ${ }^{2}$, Tao $\mathrm{Li}^{1,2}$ and Tianli Feng ${ }^{1, *}$ \\ 1 China Key Laboratory of Laser \& Infrared System (Ministry of Education), Shandong Provincial Key \\ Laboratory of Laser Technology and Application, School of Information Science and Engineering, Shandong \\ University, Qingdao 266237, China; 201920403@mail.sdu.edu.cn (J.S.); yizhou.liu@sdu.edu.cn (Y.L.); \\ shengzhi_zhao@sdu.edu.cn (S.Z.); litao@sdu.edu.cn (T.L.) \\ 2 Collaborative Innovation Center of Light Manipulations and Applications, School of Physics and Electronics, \\ Shandong Normal University, Jinan 250358, China; yuefengzhao@sdnu.edu.cn (Y.Z.); \\ yzsong@sdnu.edu.cn (Y.S.) \\ * Correspondence: tlfeng@sdu.edu.cn
}

check for updates

Citation: Shang, J.; Liu, Y.; Zhao, S.; Zhao, Y.; Song, Y.; Li, T.; Feng, T. The Investigation on Ultrafast Pulse Formation in a Tm-Ho-Codoped Mode-Locking Fiber Oscillator. Molecules 2021, 26, 3460. https:// doi.org/10.3390/molecules 26113460

Academic Editor: David Lee Phillips

Received: 2 May 2021

Accepted: 2 June 2021

Published: 7 June 2021

Publisher's Note: MDPI stays neutral with regard to jurisdictional claims in published maps and institutional affiliations.

Copyright: (c) 2021 by the authors. Licensee MDPI, Basel, Switzerland. This article is an open access article distributed under the terms and conditions of the Creative Commons Attribution (CC BY) license (https:// creativecommons.org/licenses/by/ $4.0 /)$.

\begin{abstract}
We experimentally investigate the formation of various pulses from a thulium-holmium (Tm-Ho)-codoped nonlinear polarization rotation (NPR) mode-locking fiber oscillator. The ultrafast fiber oscillator can simultaneously operate in the noise-like and soliton mode-locking regimes with two different emission wavelengths located around 1947 and $2010 \mathrm{~nm}$, which are believed to be induced from the laser transition of $\mathrm{Tm}^{3+}$ and $\mathrm{Ho}^{3+}$ ions respectively. When the noise-like pulse (NLP) and soliton pulse (SP) co-exist inside the laser oscillator, a maximum output power of $295 \mathrm{~mW}$ is achieved with a pulse repetition rate of $19.85-\mathrm{MHz}$, corresponding to a total single pulse energy of $14.86 \mathrm{~nJ}$. By adjusting the wave plates, the fiber oscillator could also deliver the dual-NLPs or dual-SPs at dual wavelengths, or single NLP and single SP at one wavelength. The highest 61-order harmonic soliton pulse and 33.4-nJ-NLP are also realized respectively with proper design of the fiber cavity.
\end{abstract}

Keywords: nonlinear polarization rotation; mode-locking; ultrafast fiber oscillator; soliton pulse; noise-like pulse; Tm-Ho-codoped fiber

\section{Introduction}

With the rapid development of ultrafast fiber lasers, increasingly complex ultrafast dynamics are discovered. The investigation of different ultrafast dynamics not only helps toward better understanding of the pulse evolution in an optical fiber, but also is useful for designing a mode-locking oscillator. Various ultrafast pulse evolution dynamics can be investigated theoretically based on the nonlinear Schrödinger equation [1,2], the coupled/complex Ginzburg-Landau equations [3,4], the Hirota bilinear formula [5], the Bogoyavlenskii-Schiff equation [6], and the Hirota-Satsuma-Ito equation [7]. The formation of soliton pulse is a well-known ultrafast dynamics which arises from the balance between the optical nonlinearity and anomalous chromatic dispersion [8-11]. The soliton pulse (SP) maintains its shape in both temporal (ps or fs) and spectral domain when propagating inside the fiber oscillator. The symmetrical Kelly sidebands distributed on the emission spectrum is a typical characteristic of the soliton pulse (see Figure 1a). In comparison, dissipative soliton is usually realized in the normal dispersion regime, always featured with a ps-long Gauss or sech shape in time domain and a square spectrum shape in frequency domain (see Figure 1b) [12-14]. Both of these two basic soliton pulses, SP and dissipative soliton, can turn into the dissipative soliton resonance (DSR) if large identical dispersion and high gain are simultaneously introduced into the mode-locking oscillator [15-18]. The DSR is characterized with a square pulse shape with an ns- or ps-pulse duration, smooth spectrum profile and large pulse energy (see Figure 1c). Furthermore, the basic soliton pulse will break into multiple pulses when the intracavity nonlinearity 
is overdriven by a large energy pulse. According to the pulse profiles in time domain, the pulse can be divided into different types: noise-like pulse (NLP) [19-23], bunched soliton pulses/optical soliton molecules [24-27] and soliton rain [28], et.al. NLP consists of a large number of small pulses randomly underlying in the same pulse envelope. Its spectrum is smooth without any spikes or modulations (see Figure 1d). Bunched soliton pulses also referred as optical soliton molecules are formed by multiple pulses gathered equal temporal distance. The typical characteristic of bunched soliton pulse is the interference fringes on the top of the spectrum (see Figure 1e). Soliton rain comprises three parts: a high peak pulse called condensed soliton phase similar to NLP with a group of multiple pulses under the envelope, the drifting pulses named drifting solitons emerging from the noise background and vanishing until reaching the condensed soliton phase, a wide noise background which manifests its existence as a small peak on the top of the spectrum in the frequency domain (see Figure 1f). These different types of pulses (soliton pulse, DSR, NLP, bunched soliton pulses, etc.) can exist in the harmonic mode-locking regime, in which the pulse reproduces itself with a multiplication of fundamental pulse repetition rate, further forming harmonic solitons [29], harmonic dissipative solitons [13], harmonic bunched solitons [27] and harmonic NLP [30]. Attractively, these pulses also can co-exist in a same fiber oscillator, which greatly enriches the ultrafast dynamics in a mode-locking laser. For example, near the zero-dispersion wavelength region of the glass fiber, two different SPs with non-equal pulse intensity are observed in an Er-doped fiber oscillator [31]. Additionally, in the Er-doped fiber oscillator, harmonic bunched-solitons and NLP are simultaneously achieved with a high nonlinear fiber [25]. On the other hand, fiber oscillators operating above the zero-dispersion wavelength region can provide a natural anomalous dispersion environment. These fiber oscillators including $2 \mu \mathrm{m}$ thulium (Tm)-doped, holmium (Ho)-doped, or Tm-Ho-codoped fiber oscillators provide another platforms for the investigation of pulse evolution dynamics [32-35]. The large gain of the Tm-doped fiber mainly is located in the $<2000 \mathrm{~nm}$ wavelength region, while with the assistance of $\mathrm{Ho}^{3+}$ ion, the large net-gain can be extended easily to the $>2000 \mathrm{~nm}$ wavelength region in a Tm-Ho-codoped fiber, resulting in a broadband wavelength emission ranging from $1.7 \mu \mathrm{m}$ to $2.1 \mu \mathrm{m}$. Besides that, the dual ions doped ultrafast fiber laser can provide more abundant pulse dynamics due to the interaction between co-doped ions. In this work, we fist report the coexistence phenomenon of NLP and SP in a nonlinear polarization rotation (NPR) mode-locking Tm-Ho-codoped fiber oscillator. The harmonic soliton pulse and NLP also can be obtained separately with proper design of the fiber cavity. In the co-existence regime, a maximum average output power of $295 \mathrm{~mW}$ is realized with a pulse repetition rate of $19.85 \mathrm{MHz}$, resulting in a pulse energy of $14.86 \mathrm{~nJ}$. The dual-NLPs or SPs at two different wavelengths, or single NLP and SP at one wavelength, are also obtained respectively by adjusting the wave plates. Moreover, harmonic soliton mode-locking with 61-order pulse is also realized by increasing cavity length. The physical formation mechanism for the coexistence of different mode-locking pulses is analyzed.

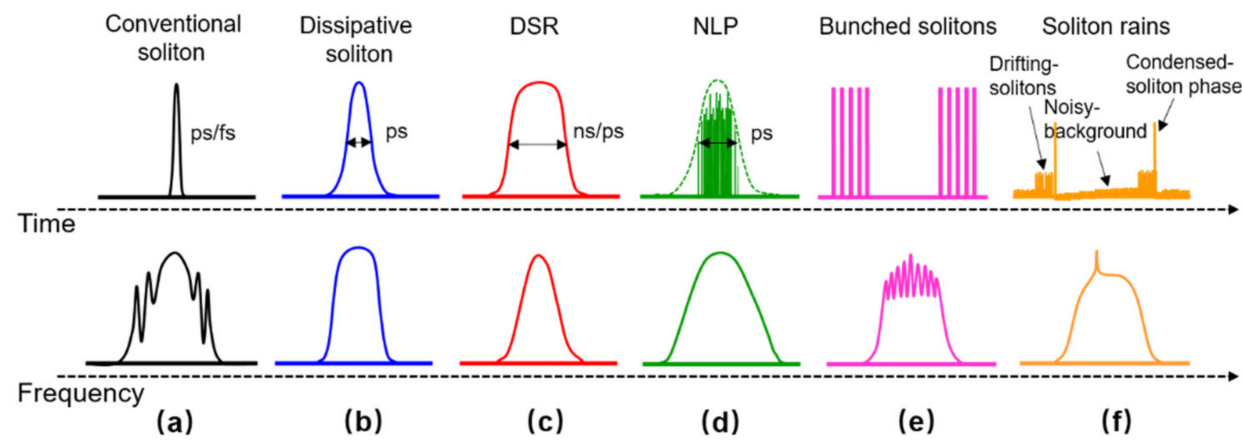

Figure 1. The typical pulse shapes and spectral profiles for conventional soliton (a), dissipative soliton (b), dissipative soliton resonance (DSR) (c), noise-like pulse (NLP) (d), bunched solitons (e), and soliton rains (f). 


\section{Results and Discussion}

The NLP and SP coexisted mode-locking operation is realized by carefully adjusting wave plates at the pump power above $1.2 \mathrm{~W}$. The power performance is recorded as Figure 2a. As the pump power scales up, the fiber laser gradually evolves from the continuous wave $(\mathrm{cw})$ regime to the $\mathrm{Q}$-switched mode-locking $(\mathrm{QML})$ regime and finally to the dual-pulse coexisted mode-locking regime. The maximum average output power reaches to $292 \mathrm{~mW}$ at the pump power of $4.23 \mathrm{~W}$. Figure $2 \mathrm{~b}$ shows the spectrum of NLP locates at a short wavelength $(\sim 1947 \mathrm{~nm})$ and possesses a smooth profile with a bandwidth of $\sim 22 \mathrm{~nm}$. The small spikes riding on the NLP spectrum are attributed to the absorption of water vapor in air. The long emission spectrum, which is the spectrum of SP verified by the symmetrically distributed Kelly sidebands, locates around $\sim 2010 \mathrm{~nm}$ with a spectral bandwidth of about $5 \mathrm{~nm}$. The typical mode-locking pulse train is shown as inset of Figure $2 \mathrm{~b}$, giving a pulse-to-pulse fluctuation of about $12 \%$, which is deteriorated by the instable NLP mode-locking. For further estimating the stability of the mode-locking operation, the radio frequency (RF) spectrum is measured for different scanning ranges shown in Figure 2c. The RF spectrum of SP is overwhelmed by the RF spectrum of NLP, which possesses a wide width and two sidelobes at the bottom of the fundamental frequency. The fundamental frequency is $19.85 \mathrm{MHz}$ with a signal-to-noise ratio (SNR) of $70 \mathrm{~dB}$, matching well with the fiber cavity length. In a broad RF spectrum range up to $2 \mathrm{GHz}$, the RF spectrum (inset of Figure 2c) shows a broad comb of harmonics with a SNR higher than $40 \mathrm{~dB}$. The pulse auto-correlation trace is also featured with the characteristic of the NLP, which consists of a narrow femtosecond spike and a hundred picoseconds pedestal (Figure 2d) [22,30]. The cross-section of the pedestal in the auto-correlation trace increases as the pump power scales up, implying the simultaneous increasing of the pulse energy of NLP.
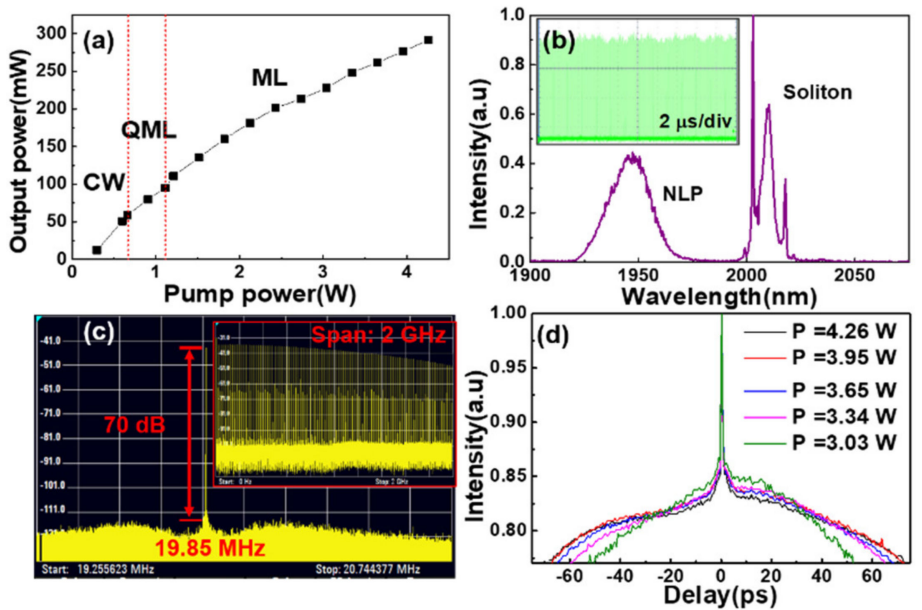

Figure 2. (a) Output power versus pump power. cw: continuous wave, QML: Q-switched modelocking, ML: mode-locking. (b) Output laser spectrum in the NLP and SP coexisted mode-locking regime. Inset: typical mode-locking pulse train at the time scale of $20 \mu \mathrm{s}$. (c) The radio frequency spectral at the scanning ranges of $1.5 \mathrm{MHz}$. Inset: $2 \mathrm{GHz}$. (d) The pulse auto-correlation traces under different pump powers, $\mathrm{P}$ : pump power.

In order to separately investigate the SP, a filter is utilized to move away the NLP in the short wavelength region $(<2000 \mathrm{~nm})$. The performances of the SP are shown in Figure 3. After the filter, the intensity of NLP is reduced remarkably, but the intensity of SP is almost unchanged relatively (see Figure 3a). Figure $3 \mathrm{~b}$ shows the measured SP trains at the time scales of $2 \mu \mathrm{s}$ and $20 \mu \mathrm{s}$. The pulse-to-pulse fluctuation is reduced from $12 \%$ (see Figure $2 b$ ) to $5 \%$. The RF spectrum in Figure 3c shows the SNR of fundamental frequency is $49 \mathrm{~dB}$ and there is no obvious NLP induced sidelobes. The inset of Figure $3 \mathrm{c}$ indicates the SNR of the harmonic combs is still larger than $20 \mathrm{~dB}$ at the $2 \mathrm{GHz}$ scanning range. The measured SP auto-correlation trace shown in Figure $3 \mathrm{~d}$ has a pedestal with a 
$\sim 20$ ps duration, arising from the residual NLP (see Figure 3a). Assuming a sech ${ }^{2}$ pulse shape, the pulse duration is determined to be 1 ps (inset of Figure $3 \mathrm{~d}$ ). The time-bandwidth product is evaluated to be 0.353 , approaching to the Fourier transformation limited value of 0.315 . By carefully rotating wave plates at the maximum pump power, the SPs at one wavelength (1966.6 or $2003.5 \mathrm{~nm}$ ) or dual-wavelengths (1933.1 and $2004.1 \mathrm{~nm}$ ), NLPs at one wavelength $(1990.0 \mathrm{~nm})$ or dual-wavelengths $(1950.4$ and $2006.5 \mathrm{~nm})$, and the coexisted SP and NLP at dual wavelengths (1937.2 and $1998.0 \mathrm{~nm}$ ) are also observed as shown in Figure 4. The wavelength spacing of the dual center wavelengths is always around $60 \mathrm{~nm}$ in different mode-locking regimes. Among these regimes, the maximum average output power of $512 \mathrm{~mW}$ is realized for the single NLP mode-locking at $1990.0 \mathrm{~nm}$, resulting in a pulse energy of $25.8 \mathrm{~nJ}$.
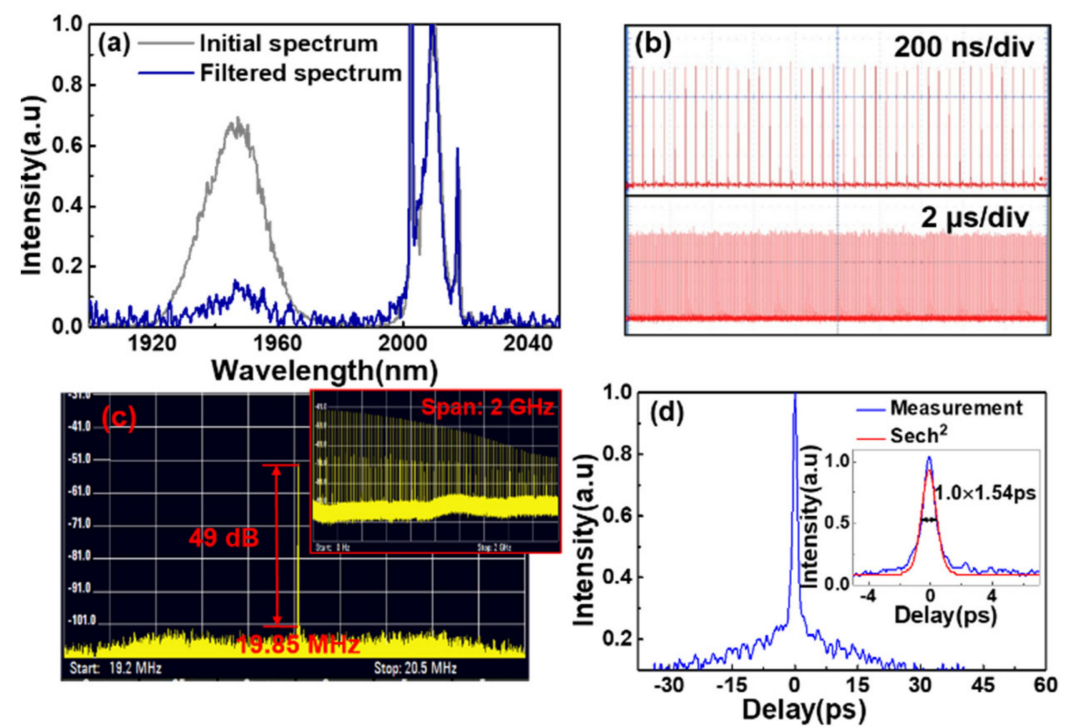

Figure 3. (a) The initial spectrum (gray line) and filtered spectrum (blue line). (b) The SP trains at the time scales of 2 and $20 \mu \mathrm{s}$. (c) The RF spectrum of SP at the scanning ranges of $1.3 \mathrm{MHz}$ and $2 \mathrm{GHz}$ (inset). (d) The SP auto-correlation trace. Inset: the SP auto-correlation trace fitted by the sech ${ }^{2}$ function.

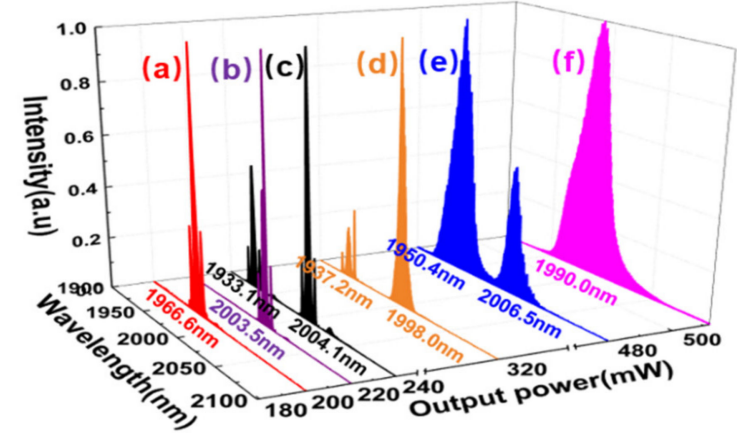

Figure 4. The output spectrum under different average output powers evolutions at the maximum pump power. The number marks the central wavelength of the emission spectrum. Single wavelength for single SP (a), (b) and single NLP (f), dual-wavelengths for dual-SPs (c) and dual-NLPs (e), and the coexisted SP and NLP (d).

By slightly changing the parameter of the laser cavity with increasing the cavity length to $\sim 25.4 \mathrm{~m}$, the soliton harmonic mode-locking and NLP mode-locking are also realized, respectively. As the pump power scales up from $0.23 \mathrm{~W}$ to $4 \mathrm{~W}$, the fiber oscillator can operate in cw regime, soliton harmonic mode-locking (HML) regime and NLP modelocking regime. These different regimes can be easily distinguished from the emission spectra, which are shown in Figure 5a. In the soliton HML regime, the pulse repetition 
rate can reach to $497.15 \mathrm{MHz}$, corresponding to the 61-order of the fundamental pulse repetition rate. This is the highest soliton order compared with the previously reported Tm-Ho-codoped HML fiber oscillators. The pulse duration is 2.29 ps by assuming a sech ${ }^{2}$ pulse shape (inset of Figure 5b) and RF spectrum shows a SNR of $41 \mathrm{~dB}$ (see Figure 5c). In NLP mode-locking regime, the dual-wavelengths with a spacing also around $\sim 60 \mathrm{~nm}$ are observed, and the central wavelengths approach to that in Figure $2 \mathrm{~b}$. The broader pedestal of NLP in Figure $5 b$ indicates a much higher pulse energy $(33.4 \mathrm{~nJ})$ than that in Figure $2 \mathrm{~d}$. The RF spectrum in Figure 5d shows more obvious sidelobes at the bottom of the fundamental frequency with a SNR of $51 \mathrm{~dB}$. It should be noted that the limited resolution of the instruments for charactering ultrafast pulses, the output instability of the fiber oscillator itself, and the environmental fluctuations can result in some uncertainties for the measured ultrafast pulse performances. In addition, the phase noise uncertainty can be precisely measured as that investigated in Reference [36].
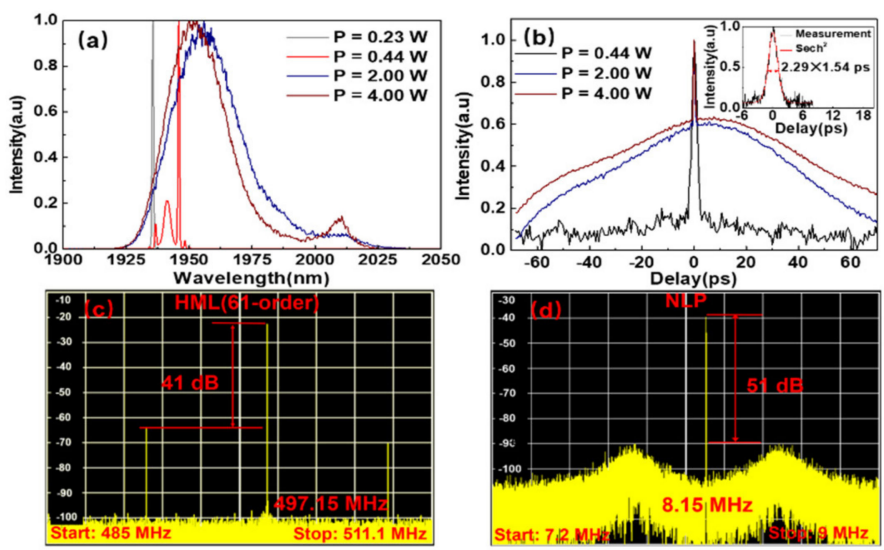

Figure 5. (a) The emission spectra under different pump powers. (b) The measured autocorrelation traces under different pump powers. Inset: the SP auto-correlation trace fitted by the sech ${ }^{2}$ function at 61-order HML regime. The RF spectra of the 61-order soliton (c) and the NLP (d) at the scanning range of $26.1 \mathrm{MHz}$ and 1.8 MHz. HML: harmonic mode-locking.

In the experiment, dual wavelength operations are always achieved in different modelocking regimes. The dual wavelength operation could arise from the spectral filter effect in the birefringent fiber or the emissions of $\mathrm{Tm}^{3+}$ and $\mathrm{Ho}^{3+}$ ions in the Tm-Ho-codoped active fiber. The period of spectra filter $\Delta \lambda$ induced by birefringence can be expressed as [37]:

$$
\Delta \lambda=\frac{\lambda^{2}}{L B_{m}+n_{2} P L \cos (2 \theta) / A_{e f f}}
$$

where $\lambda$ is the emission wavelength, $L$ is the length of birefringent fiber, $B_{m}=n_{x}-n_{y}$ is the modal birefringence, $n_{x}$ and $n_{y}$ are the refractive index for different polarizations, $n_{2}$ is the nonlinear refractive index, $P$ is the instantaneous power of the laser, $\theta$ is the angle depending on the rotation of wave-plates, and $A_{\text {eff }}$ is the effective mode area. In the experiment, the SMF can function as the birefringent fiber as Reference [37] so that the calculated modal birefringence $B_{m}$ is $4.9 \times 10^{-6}$ and the nonlinear refractive index $n_{2}$ is $2.7 \times 10^{-20} \mathrm{~m}^{2} / \mathrm{W}$ [37]. According to the experimental results, we set $\lambda=1950 \mathrm{~nm}, P=0.1 \mathrm{~W}, A_{\text {eff }}=254 \mu \mathrm{m}^{2}$, and $\Delta \lambda$ $\approx 60 \mathrm{~nm}$, while a proper $\theta$ is unable to be solved with the experimental cavity length $L$ of $10.4 \mathrm{~m}$ or $25.4 \mathrm{~m}$. Therefore, we believe that the dual-wavelength emission is independent of the spectral filter effect in the SMF-based birefringent fiber. Moreover, we find that the dual-wavelength emission only is observed when the pump power exceeds a certain value as shown in Figure 5. This is a main characteristic of Tm-Ho-co-doped laser which requires a high pump power for energy transfer between $\mathrm{Tm}^{3+}$ and $\mathrm{Ho}^{3+}$ ions to emit dual wavelengths. The formation mechanism for dual-wavelength emission is different from those reported methods [30,38-44]. 
The emission and absorption spectra of $\mathrm{Tm}^{3+}$ and $\mathrm{Ho}^{3+}$ ions are shown in Figure 6a. Although the gain wavelength region of the $\mathrm{Tm}^{3+}$ ion is partly overlapped with the absorption of $\mathrm{Ho}^{3+}$ ion, there still exists net gain in the wavelength region below $2000 \mathrm{~nm}$. With the assistance of $\mathrm{Ho}^{3+}$ ion, the large net-gain can be extended to above 2000-nm wavelength region in a Tm-Ho-codoped system. The ion transition processes in the Tm-Ho-codoped active fiber are simplified as shown in Figure $6 \mathrm{~b}$. The $\mathrm{Tm}^{3+}$ ions at the ground state of ${ }^{3} \mathrm{H}_{6}$ are excited to the upper energy level ${ }^{3} \mathrm{~F}_{4}$ by the $1560-\mathrm{nm}$ pumping laser. When the pump power is low, most of the $\mathrm{Tm}^{3+}$ ions at the energy level of ${ }^{3} \mathrm{~F}_{4}$ will return to the ${ }^{3} \mathrm{H}_{6}$ accompanied by the laser emission in the short wavelength region below $2000 \mathrm{~nm}$ (laser emission 1 in Figure 6b). The energy transition between ${ }^{3} \mathrm{~F}_{4}$ in $\mathrm{Tm}^{3+}$ ion and ${ }^{5} \mathrm{I}_{7}$ in $\mathrm{Ho}^{3+}$ ion can be ignored so that only one wavelength emission can be observed under weak pump power. As the pump power scales up, the energy level of ${ }^{3} \mathrm{~F}_{4}$ in the $\mathrm{Tm}^{3+}$ ion is strongly occupied, which results in a large energy transition between $\mathrm{Tm}^{3+}$ ion and $\mathrm{Ho}^{3+}$ ion. So other than the laser emission in short wavelength region, the transition from the energy level ${ }^{5} \mathrm{I}_{7}$ to the energy level ${ }^{5} \mathrm{I}_{8}$ of $\mathrm{Ho}^{3+}$ ions generates another laser emission above $2000 \mathrm{~nm}$ (laser emission 2 in Figure 6b). It should be noted that for the coexisted pulses at dual wavelengths, the laser emission in short wavelength region always possesses a large gain compared with that in the long wavelength region due to the low concentration of $\mathrm{Ho}^{3+}$ ions. For example, as shown in Figures $2 \mathrm{~b}, 4 \mathrm{e}$ and $5 \mathrm{a}$, under strong pump power, the SP or NLP with a low pulse energy is formed at a long wavelength via the emission transition in $\mathrm{Ho}^{3+}$ ion, while the formed pulse at short wavelength accumulates a large energy due to the large gain, which facilities the formation of large energy NLP. However, in Figure $4 \mathrm{~b}-\mathrm{d}$, we find that the SP and NLP with large energies are also formed around $2000 \mathrm{~nm}$. We believe this is attributed to the co-interaction of $\mathrm{Tm}^{3+}$ and $\mathrm{Ho}^{3+}$ ions in this wavelength region (see Figure 6a).
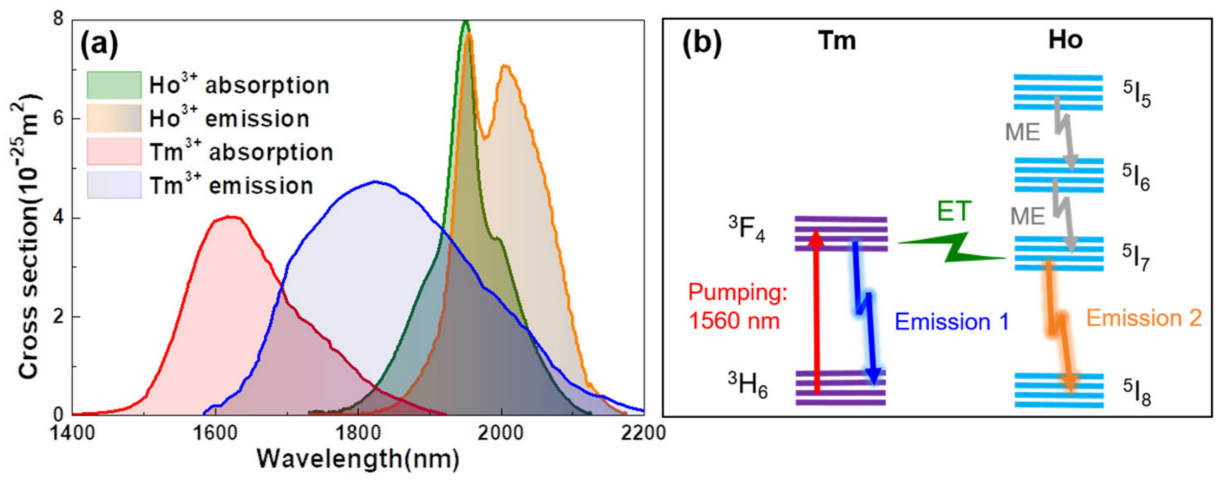

Figure 6. (a) The absorption and emission cross section of $\mathrm{Tm}^{3+}$ and $\mathrm{Ho}^{3+}$. (b) The simplified energy diagram of Tm-Ho-codoped gain fiber pumped at $1560 \mathrm{~nm}$. ET: energy transition, ME: multi-phonon emission.

\section{Materials and Methods}

The schematic diagram of the Tm-Ho-codoped mode-locking fiber oscillator is shown as Figure 7. The pump source is a continuous wave 1562-nm Er-doped fiber laser amplifier (FLA), which delivers a maximum output power of $4.23 \mathrm{~W}$ with a power instability of $0.3 \%$ measured within $60 \mathrm{~min}$. The pumping laser is guided into the Tm-Ho-codoped fiber ring cavity by a wavelength division multiplex (WDM). The fiber ring cavity consists of a $4.3 \mathrm{~m}$ long Tm-Ho-codoped single mode active fiber (Coractive, SM-TH512, $23 \mathrm{~dB} / \mathrm{m}$ at $1570 \mathrm{~nm},-56 \mathrm{ps}^{2} / \mathrm{km}$ at $1900 \mathrm{~nm}, \mathrm{CAN}$ ), a polarization independent isolator (PI-ISO), a group of NPR free-space optical components, and a $5.4 \mathrm{~m}$ long passive single-mode fiber (Nufern, SMF-28e, $-67 \mathrm{ps}^{2} / \mathrm{km}$ at $1900 \mathrm{~nm}$, USA). The NPR optical component includes two quarter-wave plates, a half-wave plate, and a polarization beam splitter (PBS). The PBS simultaneously functions as both polarizer and output coupler. Considering the pigtail fiber of all optical components inside the fiber cavity, the total length of the fiber ring cavity 
is close to $10.4 \mathrm{~m}$. The mode-locking operation can be realized by carefully adjusting the wave plates. The output pulse train is detected by an InGaAs PIN detector (EOT, ET-5000, USA) and observed with an oscilloscope (Tektronix, DPO 4102B-L, USA).

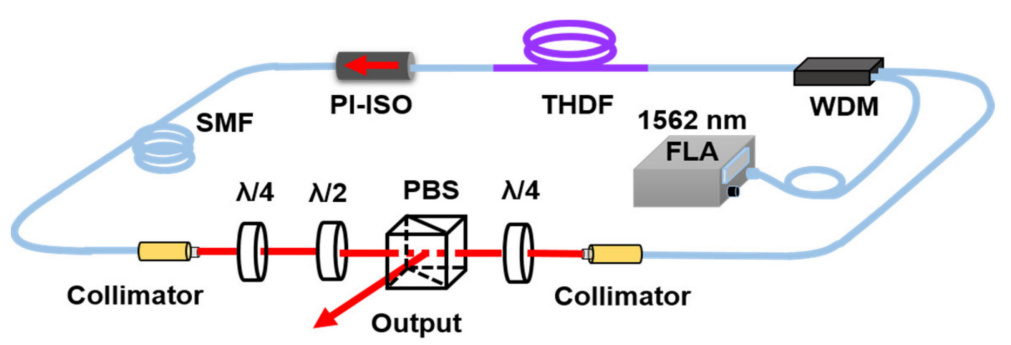

Figure 7. Schematic diagram of the Tm-Ho-codoped fiber oscillator. FLA: fiber laser amplifier; PI-ISO: polarization-independent isolator; WDM: wavelength division multiplex; THDF: Tm-Hocodoped fiber; SMF: single-mode-fiber; PBS: polarization beam splitter; $\lambda / 2$ : half-wave plate; $\lambda / 4$ : quarter-wave plate.

\section{Conclusions}

In this work, first we observe the coexisted noise-like pulse and soliton pulse in the thulium-holmium-codoped ultrafast fiber oscillator. By carefully adjusting the wave plates, the coexisted noise-like pulse and soliton pulse can involve into the formation of dualnoise-like pulses or dual-soliton pulses at dual-wavelengths and single noise-like pulse or single soliton pulse at one wavelength. A 61-order harmonic soliton pulse and the 33.4nJ-noise-like pulse are also realized respectively by prolonging the fiber oscillator length We believe the dual-wavelength emissions are attributed to the transitions of $\mathrm{Tm}^{3+}$ and $\mathrm{Ho}^{3+}$ ions respectively of the gain fiber. The coexisted NLP and SP at different wavelengths depend on the different gain under a strong pumping power.

Author Contributions: Conceptualization, T.F. and J.S.; methodology, Y.L.; software, J.S.; validation, S.Z., Y.Z. and Y.S.; formal analysis, T.F.; investigation, J.S.; resources, T.F.; data curation, T.F.; writingoriginal draft preparation, J.S.; writing—review and editing, T.F.; visualization, J.S.; supervision, T.F.; project administration, T.L.; funding acquisition, T.F. All authors have read and agreed to the published version of the manuscript.

Funding: This research was funded by the National Key Research and Development Program of China Grants 2016YFB1102201, Qilu Young Scholar Program of Shandong University, Taishan Scholar Foundation of Shandong Province Grants tsqn201812010, Natural Science Foundation of Shandong Province grants ZR2018MF033 and ZR2020QF096, and National Natural Science Foundation of China Grants 61775119 and 62005144.

Institutional Review Board Statement: Not applicable.

Informed Consent Statement: Not applicable.

Data Availability Statement: Data are contained within this article.

Acknowledgments: The authors thanks to the National Key Research and Development Program of China Grants 2016YFB1102201, Qilu Young Scholar Program of Shandong University, Taishan Scholar Foundation of Shandong Province Grants tsqn201812010, Natural Science Foundation of Shandong Province grants ZR2018MF033 and ZR2020QF096, and National Natural Science Foundation of China Grants 61775119 and 62005144 for their financial support.

Conflicts of Interest: The authors declare no conflict of interest.

Sample Availability: Samples of the patches are available from the authors. 


\section{References}

1. Ma, W.X.; Chen, M. Direct search for exact solutions to the nonlinear Schrödinger equation. Appl. Math. Comput. 2009, 215, 2835-2842. [CrossRef]

2. Chabchoub, A.; Kibler, B.; Finot, C.; Millot, G.; Onorato, M.; Dudley, J.M.; Babanin, A.V. The nonlinear Schrödinger equation and the propagation of weakly nonlinear waves in optical fibers and on the water surface. Ann. Phys. 2015, 361, 490. [CrossRef]

3. Kobtsev, S.M.; Kukarin, S.V.; Smirnov, S.V.; Fedotov, Y.S. High-energy mode-locked all-fiber laser with ultralong resonator. Laser Phys. 2010, 20, 351-356. [CrossRef]

4. Tang, D.; Zhao, L.; Zhao, B. Soliton collapse and bunched noise-like pulse generation in a passively mode-locked fiber ring laser. Opt. Express 2005, 13, 2289-2294. [CrossRef]

5. Ma, W.X. N-soliton solutions and the Hirota conditions in (2+1)-dimensions. Opt. Quant. Electron. 2020, 52, 511. [CrossRef]

6. Yu, S.J.; Toda, K.; Sasa, K.; Fukuyama, T. N soliton solutions to the Bogoyavlenskii-Schiff equation and a quest for the soliton solution in (3+1) dimensions. J. Phys. A Math. Gen. 1998, 31, 3337. [CrossRef]

7. Zhou, Y.; Manukure, S.; Ma, W.X. Lump and lump-soliton solutions to the Hirota-Satsuma-Ito equation. Commun. Nonlinear Sci. Numer. Simulat. 2019, 68, 56-62. [CrossRef]

8. Mitschke, F.M.; Mollenauer, L.F. Ultrashort pulses from the soliton laser. Opt. Lett. 1987, 12, 407-409. [CrossRef]

9. Li, J.; Wang, Y.; Luo, H.; Liu, Y.; Yan, Z.; Sun, Z.; Zhang, L. Kelly sideband suppression and wavelength tuning of a conventional soliton in a Tm-doped hybrid mode-locked fiber laser with an all-fiber Lyot filter. Photon. Res. 2019, 7, 103-109. [CrossRef]

10. Wang, M.; Wei, R.; Zhu, Z.; Ruan, S.; Yan, P.; Wang, J.; Hasan, T.; Sun, Z. 172 fs, 24.3 kW peak power pulse generation from a Ho-doped fiber laser system. Opt. Lett. 2018, 43, 4619-4622. [CrossRef]

11. Luo, Z.; Li, Y.; Huang, Y.; Zhong, M.; Wan, X. Graphene mode-locked and Q-switched 2- $\mu \mathrm{m}$ Tm/Ho codoped fiber lasers using 1212-nm high-efficient pumping. Opt. Eng. 2016, 55, 081310. [CrossRef]

12. Grelu, P.; Akhmediev, N. Dissipative solitons for mode-locked lasers. Nat. Photonics 2012, 6, 84-92. [CrossRef]

13. Yang, N.; Tang, Y.; Xu, J. High-energy harmonic mode-locked $2 \mu \mathrm{m}$ dissipative soliton fiber lasers. Laser Phys. Lett. 2015, 12, 085102. [CrossRef]

14. Zhang, H.; Tang, D.Y.; Wu, X.; Zhao, L.M. Multi-wavelength dissipative soliton operation of an erbium-doped fiber laser. Opt. Express 2009, 17, 12692-12697. [CrossRef]

15. Chang, W.; Ankiewicz, A.; Soto-Crespo, J.M.; Akhmediev, N. Dissipative soliton resonances. Phys. Rev. A 2008, 78, 023830. [CrossRef]

16. Cheng, Z.; Li, H.; Wang, P. Simulation of generation of dissipative soliton, dissipative soliton resonance and noise-like pulse in Yb-doped mode-locked fiber lasers. Opt. Express 2015, 23, 5972-5981. [CrossRef] [PubMed]

17. Han, L.; Wang, T.; Song, G.; Ma, W. Generation of Dissipative Soliton Resonance Pulse in 2 um All-Polarization-Maintaining Mode-Locked Fiber Laser. In Proceedings of the 2020 IEEE 5th Optoelectronics Global Conference (OGC), Shenzhen, China, 7-11 September 2020.

18. Duan, L.; Liu, X.; Mao, D.; Wang, L.; Wang, G. Experimental observation of dissipative soliton resonance in an anomalousdispersion fiber laser. Opt. Express 2012, 20, 265-270. [CrossRef]

19. Chernykh, A.I.; Turitsyn, S.K. Soliton and collapse regimes of pulse generation in passively mode-locking laser systems. Opt. Lett. 1995, 20, 398-400. [CrossRef]

20. Jeong, Y.; Vazquez-Zuniga, L.A.; Lee, S.; Kwon, Y. On the formation of noise-like pulses in fiber ring cavity configurations. Opt. Fiber Technol. 2014, 20, 575-592. [CrossRef]

21. Zhao, L.M.; Tang, D.Y.; Wu, J.; Fu, X.Q.; Wen, S.C. Noise-like pulse in a gain-guided soliton fiber laser. Opt. Express 2007, 15, 2145-2150. [CrossRef]

22. Ahmad, H.; Ahmed, M.H.M.; Samion, M.Z. Generation of mode-locking noise-like pulses in double-clad Tm-doped fibre laser with nonlinear optical loop mirror. J. Mod. Optic. 2020, 67, 146-152. [CrossRef]

23. Zhang, J.; Li, Z.; Sun, Z.; Luo, H.; Liu, Y.; Yan, Z.; Mou, C.; Zhang, L.; Turitsyn, S.K. All-fiber Passively Mode-locking Tm-Doped NOLM-Based Oscillator Operating at 2- $\mu \mathrm{m}$ in Both Soliton and Noisy-Pulse Regimes. Opt. Express 2014, $22,7875-7882$.

24. Malomed, B.A. Bound solitons in coupled nonlinear Schrödinger equations. Phys. Rev. A 1992, 45, R8321-R8323. [CrossRef] [PubMed]

25. Huang, Y.Q.; Hu, Z.A.; Cui, H.; Luo, Z.C.; Luo, A.P.; Xu, W.C. Coexistence of harmonic soliton molecules and rectangular noise-like pulses in a figure-eight fiber laser. Opt. Lett. 2016, 41, 4056-4059. [CrossRef] [PubMed]

26. Kokhanovskiy, A.; Kuprikov, E.; Ivanenko, A.; Kobtsev, S. All-polarisation-maintaining modified figure-of-8 fibre laser as a source of soliton molecules. Laser Phys. Lett. 2020, 17, 085101. [CrossRef]

27. Luo, W.; Liu, X.; Li, X.; Li, S.; Xu, W.; Wang, L.; Shi, Z.; Zhang, C. SnSe 2 realizes soliton rain and harmonic soliton molecules in erbium-doped fiber lasers. Nanotechnology 2021, 32, 165203. [CrossRef]

28. Chouli, S.; Grelu, P. Rains of solitons in a fiber laser. Opt. Express 2009, 17, 11776-11781. [CrossRef]

29. Akhmediev, N.; Soto-Crespo, J.M.; Town, G. Pulsating solitons, chaotic solitons, period doubling, and pulse coexistence in mode-locked lasers: Complex Ginzburg-Landau equation approach. Phys. Rev. E 2001, 63, 056602. [CrossRef]

30. Wang, Y.; Li, J.; Zhang, E.; Mo, K.; Wang, Y.; Liu, F.; Zhou, X.; Liu, Y. Coexistence of noise-like pulse and high repetition rate harmonic mode-locking in a dual-wavelength mode-locked Tm-doped fiber laser. Opt. Express 2017, 25, 17192-17200. [CrossRef] 
31. Liu, X. Coexistence of strong and weak pulses in a fiber laser with largely anomalous dispersion. Opt. Express 2011, $19,5874-5887$. [CrossRef]

32. Esterowitz, L. Diode pumped holmium, thulium and erbium lasers between 2 and 3 micrometers operating $\mathrm{cw}$ at room temperature. Opt. Eng. 1990, 29, 676-680. [CrossRef]

33. Hanna, D.C.; Jauncey, I.M.; Percival, R.M.; Perry, I.R.; Smart, R.G.; Suni, P.J.; Townsend, J.E.; Tropper, A.C. Continuous-wave oscillation of a monomode thulium-doped fibre laser. Electron. Lett. 1988, 24, 1222-1223. [CrossRef]

34. Kadel, R.; Washburn, B.R. All-fiber passively mode-locked thulium/holmium laser with two center wavelengths. Appl. Opt. 2012, 51, 6465-6470. [CrossRef]

35. Filatova, S.A.; Kamynin, V.A.; Arutyunyan, N.R.; Pozharov, A.S.; Obraztsova, E.D.; Itrin, P.A.; Tsvetkov, V.B. Comparison of mode-locking regimes in a holmium fibre laser. Quantum Electron. 2018, 48, 1113-1117. [CrossRef]

36. Salzenstein, P.; Pavlyuchenko, E. Uncertainty Evaluation on a $10.52 \mathrm{GHz}(5 \mathrm{dBm})$ Optoelectronic Oscillator Phase Noise Performance. Micromachines 2021, 12, 474. [CrossRef]

37. Yan, Z.; Li, X.; Tang, Y.; Shum, P.P.; Yu, X.; Zhang, Y.; Wang, Q.J. Tunable and switchable dual-wavelength Tm-doped mode-locked fiber laser by nonlinear polarization evolution. Opt. Express 2015, 23, 4369-4376. [CrossRef] [PubMed]

38. Yan, Z.; Sun, B.; Li, X.; Luo, J.; Shum, P.P.; Yu, X.; Zhang, Y.; Wang, Q. Widely tunable Tm-doped mode-locked all-fiber laser. Sci. Rep. 2016, 6, 27245. [CrossRef] [PubMed]

39. Yan, Z.; Tang, Y.; Sun, B.; Liu, T.; Li, X.; Ping, P.S.; Yu, X.; Zhang, Y.; Wang, Q.J. Switchable multi-wavelength Tm-doped mode-locked fiber laser. Opt. Lett. 2015, 40, 1916-1919. [CrossRef]

40. Wang, Y.; Li, J.; Zhai, B.; Hu, Y.; Mo, K.; Lu, R.; Liu, Y. Tunable and switchable dual-wavelength mode-locked Tm ${ }^{3+}$-doped fiber laser based on a fiber taper. Opt. Express 2016, 24, 15299-15306. [CrossRef]

41. Zhao, N.; Liu, M.; Liu, H.; Zheng, X.W.; Ning, Q.Y.; Luo, A.P.; Luo, Z.C.; Xu, W.C. Dual-wavelength rectangular pulse Yb-doped fiber laser using a microfiber-based graphene saturable absorber. Opt. Express 2014, 22, 10906-10913. [CrossRef] [PubMed]

42. Feng, X.; Tam, H.; Wai, P.K.A. Stable and uniform multi wavelength erbium-doped fiber laser using nonlinear polarization rotation. Opt. Express 2006, 14, 8205-8210. [CrossRef] [PubMed]

43. Sun, B.; Luo, J.; Yan, Z.; Liu, K.; Ji, J.; Zhang, Y.; Wang, Q.J.; Yu, X. 1867-2010 nm tunable femtosecond thulium-doped all-fiber laser. Opt. Express 2017, 25, 8997-9002. [CrossRef] [PubMed]

44. Talaverano, L.; Abad, S.; Jarabo, S.; Lopez-Amo, M. Multiwavelength fiber laser sources with Bragg-grating sensor multiplexing capability. J. Lightwave Technol. 2001, 19, 553-558. [CrossRef] 